\title{
Drugs affecting the renin-angiotensin system and survival from cancer: a population based study of breast, colorectal and prostate cancer patient cohorts
}

Chris R Cardwell ${ }^{1 *}$, Úna C Mc Menamin', Blánaid M Hicks', Carmel Hughes², Marie M Cantwell ${ }^{1}$ and Liam J Murray ${ }^{1}$

\begin{abstract}
Background: Angiotensin-converting enzyme inhibitors (ACEls) and angiotensin II receptor blockers (ARBs) are commonly prescribed to the growing number of cancer patients (more than two million in the UK alone) often to treat hypertension. However, increased fatal cancer in ARB users in a randomized trial and increased breast cancer recurrence rates in ACEI users in a recent observational study have raised concerns about their safety in cancer patients. We investigated whether ACEI or ARB use after breast, colorectal or prostate cancer diagnosis was associated with increased risk of cancer-specific mortality.

Methods: Population-based cohorts of 9,814 breast, 4,762 colorectal and 6,339 prostate cancer patients newly diagnosed from 1998 to 2006 were identified in the UK Clinical Practice Research Datalink and confirmed by cancer registry linkage. Cancer-specific and all-cause mortality were identified from Office of National Statistics mortality data in 2011 (allowing up to 13 years of follow-up). A nested case-control analysis was conducted to compare ACEI/ARB use (from general practitioner prescription records) in cancer patients dying from cancer with up to five controls (not dying from cancer). Conditional logistic regression estimated the risk of cancer-specific, and all-cause, death in ACEI/ARB users compared with non-users.

Results: The main analysis included 1,435 breast, 1,511 colorectal and 1,184 prostate cancer-specific deaths (and 7,106 breast, 7,291 colorectal and 5,849 prostate cancer controls). There was no increase in cancer-specific mortality in patients using ARBs after diagnosis of breast (adjusted odds ratio (OR) $=1.0695 \%$ confidence interval (Cl) $0.84,1.35$ ), colorectal (adjusted $\mathrm{OR}=0.8295 \% \mathrm{Cl} 0.64,1.07$ ) or prostate cancer (adjusted OR $=0.7995 \% \mathrm{Cl} 0.61,1.03$ ). There was also no evidence of increases in cancer-specific mortality with ACEl use for breast (adjusted OR $=1.0695 \% \mathrm{Cl}$ 0.89, 1.27), colorectal (adjusted $\mathrm{OR}=0.7895 \% \mathrm{Cl} 0.66,0.92$ ) or prostate cancer (adjusted $\mathrm{OR}=0.7895 \% \mathrm{Cl} 0.66,0.92$ ).

Conclusions: Overall, we found no evidence of increased risks of cancer-specific mortality in breast, colorectal or prostate cancer patients who used ACEI or ARBs after diagnosis. These results provide some reassurance that these medications are safe in patients diagnosed with these cancers.
\end{abstract}

Keywords: Colorectal cancer, Breast cancer, Prostate cancer, Mortality, Angiotensin-converting enzyme inhibitors and angiotensin II receptor blockers

\footnotetext{
* Correspondence: c.cardwell@qub.ac.uk

${ }^{1}$ Cancer Epidemiology and Health Services Research Group, Centre for Public

Health, Queen's University Belfast, Belfast, Northern Ireland

Full list of author information is available at the end of the article
} 


\section{Background}

Angiotensin-converting enzyme inhibitors (ACEIs) and angiotensin II receptor blockers (ARBs) are commonly prescribed to the large and growing number of individuals with cancer (for example, currently more than two million in the UK [1] and 13 million in the US [2]) often to treat hypertension which affects around $40 \%$ of cancer patients [3]. However, the possible effect of ACEIs and ARBs on cancer is subject to much debate. Concerns were first raised in 2003 when the Candesartan in Heart Failure Assessment of Reduction in Mortality and Morbidity (CHARM) trial [4] unexpectedly reported a significant $40 \%$ increase in fatal cancer in patients randomized to candesartan (an ARB) compared with placebo (relative risk $(\mathrm{RR})=1.42$ 95\% CI $1.02,1.98 ; P=0.04$ ). In part conducted because of this finding, a 2010 meta-analysis of randomized trials observed increased cancer risk with ARBs [5]. Preclinical studies also suggested a biological rationale for an increase in cancer risk in ARB users [6,7]. A later meta-analysis observed no association for ARBs but observed a small increased risk of cancer in combination ARB and ACEI users [8]. These findings for cancer risk were further investigated in large population-based observational studies [9-12] which, although generally negative, observed small increases in cancer risk, for instance, for breast and prostate cancer in ARB users [12] and lung cancer in ACEI users [11].

These results, and particularly the increases in fatal cancer observed in the 2003 ARB trial [4], raise questions about the safety of ACEI and ARB use in cancer patients. Despite these findings, there have not been any studies which have investigated ARB use separately after cancer diagnosis and cancer progression in prostate or colorectal cancer patients and only two studies have investigated the specific association between ARB use and cancer progression in breast cancer patients [13,14]. ACEIs and cancer progression has also received little attention with only two studies of ACEI use in colorectal cancer patients [15,16] and one in prostate cancer patients [17]. Five studies have investigated ACEI use in breast cancer patients and cancer progression but have reached conflicting results [13,14,18-20]. Further investigation of ACEIs and ARBs and cancer progression is, therefore, warranted.

This study investigated whether ACEI or ARB use after diagnosis of breast, colorectal or prostate cancer was associated with increased cancer-specific, or all cause, mortality in large population-based cohorts of cancer patients.

\section{Methods}

\section{Study design}

Cohort studies were conducted utilizing linkages between the English National Cancer Data Repository (NCDR), the UK Clinical Practice Research Datalink (CPRD) and the Office of National Statistics (ONS) death registrations. The NCDR data include date and site of primary cancer diagnosis and clinical data, such as stage and treatment. The CPRD is the world's largest database of longitudinal patient records comprising around $8 \%$ of the UK population and includes demographic information, clinical diagnoses and prescription data which is of documented high quality [21,22]. Ethical approval for all observational research using CPRD data has been obtained from a multicenter research ethics committee [23]. Linkages between the datasets were conducted using a deterministic algorithm based upon National Health Service (NHS) number, gender, date of birth and postcode. Patients were included in the cohorts if they had a CPRD diagnosis code for breast (women only), colorectal or prostate cancer which was confirmed by NCDR cancer diagnosis (based upon International Classification of Diseases (ICD) codes of C50 for breast cancer, C61 for prostate cancer, and C18, C19 and C20 for colorectal cancer) from 1998 to 2006. Cancer patients with previous NCDR cancer diagnosis, apart from in situ neoplasms and non-melanoma skin cancers, were excluded. Cancer patients were also excluded if the date of cancer diagnosis preceded CPRD research quality records. Date and cause of death up to 2011 were taken from ONS. Analysis was restricted to individuals with available ONS mortality data from cancer diagnosis.

\section{ACEI\ARB identification}

ACEIs and ARBs were defined as all agents within the two drug classes according to the British National Formulary [24] (BNF, chapters 2.5.5.1 and 2.5.5.2, respectively). ACEI and ARB prescriptions within the cohorts from CPRD prescribing data were counted and converted to daily defined doses (DDD) on the basis of the quantity and strength (as defined by the World Health Organization [25]). A quantity of 28 tablets was assumed for approximately $2 \%$ of prescriptions where quantity was missing or inconsistent. Medication usage was ascertained in the exposure period described later.

\section{Potential confounders}

Data available from the NCDR included stage, histological grade, Gleason score (for prostate cancer), surgery, chemotherapy and radiotherapy in the six months after diagnosis. Gleason score was converted to grade to increase completeness [26]. General practitioner (GP) prescribing data were used to determine hormone therapy in the first six months after cancer diagnosis including androgen therapy for prostate cancer (BNF chapter 8.3.4.2, including gonadorelin analogues and anti-androgens) and tamoxifen and aromatase inhibitors for breast cancer (BNF chapter 8.3.4.1). Breast and prostate cancer patients were excluded if hormone therapy preceded cancer diagnosis by eight weeks. In breast cancer patients, hormone replacement therapy (HRT) for estrogen and progestogens (BNF chapters 6.4.1. and 6.4.2.) was determined prior to diagnosis. Low 
dose aspirin and statin use were taken from GP prescription records. Smoking, alcohol intake and body mass index (BMI) were determined from the closest GP record prior to cancer diagnosis (records older than ten years were ignored). Comorbidities were determined from GP diagnosis codes on the basis of diagnoses contributing to a recent adaptation of the Charlson comorbidity index for GPRD [27].

\section{Data analysis}

The cancer cohorts were initially analyzed using a time matched nested case-control approach, a common approach, for example [28], which accounts for immortal time bias [29] without requiring complicated statistical techniques [30] with minimal loss of precision [31], and a time varying covariate approach, described later. Breast cancer cases were members who had died due to breast cancer (with an ICD code of C50 as the underlying cause of death) and these were matched on age (in five year intervals) and year of cancer diagnosis to five risk-set controls who lived at least as long after their cancer diagnosis. Corresponding analyses were conducted for colorectal cancer cases (ICD codes of C18, C19, C20, C21 or C26 as their underlying cause of death) who were matched to risk-set controls on gender, site (colon or rectal), age (in five year intervals) and year of cancer diagnosis (in two year intervals) and prostate cancer cases (with ICD codes of C61 as their underlying cause of death) who were matched to risk set controls on age (in five year intervals) and year of cancer diagnosis.

The exposure period (for identification of post-diagnostic medication usage) in cases was the period from cancer diagnosis until six months prior to cancer-specific death. The exposure period in the controls was fixed to be the same duration as that of their matched cases and began at the date of cancer diagnosis in the control. The exposure period did not include prescriptions in the six month period prior to death as these may reflect end of life treatment or increased exposure to healthcare professionals (sensitivity analyses investigated increasing this to 12 months). Analyses were restricted to individuals with at least one year of follow-up after cancer diagnosis.

Conditional logistic regression was used to estimate the odds of death from cancer in cancer patients prescribed one or more ACEIs in the exposure period compared to those with none and corresponding odds ratios (ORs) and 95\% confidence intervals (95\% CIs) were determined before and after adjustment for potential confounders. Similar analyses were conducted by duration of medication usage (investigating individuals prescribed over 365 DDDs of ACEIs in the exposure period equivalent to one year of usage). These analyses were repeated for ARBs. An analysis of all-cause mortality was also conducted in which cancer patients who died from any-cause were matched to risk-set controls (using the same matching criteria as in the cancerspecific analyses) and conditional logistic regression models were applied as described previously. A secondary analysis was conducted to investigate pre-diagnostic ACEI/ARB use. Two sensitivity analyses were conducted which restricted the non-user group to patients with more similar indications. In one analysis, the cancer cohorts were first restricted to individuals using any antihypertensive medications in the year prior to cancer diagnosis (including diuretics, vasodilator antihypertensive drugs, centrally acting antihypertensive drugs, alpha-adrenoceptor blocking drugs, beta-blockers, ACEIs, ARBs, renin inhibitors, and calcium channel blockers) and the analysis was conducted as previously. In the other sensitivity analysis, the full cohort was used as in the main analysis but ORs were calculated comparing ACEI/ARB users in the exposure period not to ACEI/ARB non-users in the exposure period (as in the main analysis) but to ACEI/ARB non-users who had used at least one other antihypertensive medication in the exposure period. Sensitivity analyses were also conducted additionally adjusting for other antihypertensive use and in breast and prostate cancer patients, adjusting for hormone therapy at any time after diagnosis (not just in the first six months after diagnosis as in the main analysis). A sensitivity analyses was conducted in prostate cancer patients adjusting for Gleason score and in breast cancer patients restricted to cancer registries with high rates of available stage (overall, over $85 \%$ complete). Stratified analyses were conducted by stage (for colorectal and breast cancer), sex (for colorectal) and site (for colorectal cancer). Stratified analyses were conducted after re-matching cases to controls within strata. Interaction tests were used to compare associations between strata [32].

Additionally, the cancer cohort was analyzed, without conversion to case-control data, applying survival analysis to investigate ACEI/ARB exposure as a time varying covariate (individuals were considered non-users prior to use and users after a lag of six months after first use, to mimic the case-control analysis) [29]. A similar dose response analysis was conducted with individuals considered non-users prior to six months after first use, a short term user between six months after first use and six months after 365 DDDs and a longer term user after this time. A separate analysis was conducted additionally adjusting for competing risk of deaths using Fine and Gray's proportional subhazards model (not shown, as results similar) [33].

A pre-study power calculation based upon 15\% ACEI use and predicted numbers suggested the study would have over $80 \%$ power to detect an OR of 1.20 for cancerspecific mortality in patients receiving ACEIs in each of the cancer cohorts (breast, colorectal, prostate). More accurately, based upon the final numbers (shown in Table 1) and ACEI and ARB use (shown in Table 2), the study had 
Table 1 Characteristics of cancer patients who die from cancer (cases) compared with controls, by cancer site

\begin{tabular}{|c|c|c|c|c|c|c|c|c|c|}
\hline & \multicolumn{2}{|c|}{ Breast cancer } & \multirow[b]{2}{*}{$P$} & \multicolumn{2}{|c|}{ Colorectal cancer } & \multirow[b]{2}{*}{$P$} & \multicolumn{2}{|c|}{ Prostate cancer } & \multirow[b]{2}{*}{$P$} \\
\hline & $\begin{array}{c}\text { Cases } \\
\text { number (\%) }\end{array}$ & $\begin{array}{c}\text { Controls } \\
\text { number (\%) }\end{array}$ & & $\begin{array}{c}\text { Cases } \\
\text { number (\%) }\end{array}$ & $\begin{array}{c}\text { Controls } \\
\text { number (\%) }\end{array}$ & & $\begin{array}{c}\text { Cases } \\
\text { number (\%) }\end{array}$ & $\begin{array}{c}\text { Controls } \\
\text { number (\%) }\end{array}$ & \\
\hline \multicolumn{10}{|l|}{ Year of diagnosis } \\
\hline 1998 to 2000 & $500(35)$ & $2,466(35)$ & N.A. & $379(25)$ & $1,842(25)$ & N.A. & $396(33)$ & $1,941(33)$ & N.A. \\
\hline 2001 to 2003 & $518(36)$ & $2,570(36)$ & & $564(37)$ & $2,721(37)$ & & 461 (39) & 2,281 (39) & \\
\hline 2003 to 2006 & $417(29)$ & $2,070(29)$ & & $568(38)$ & $2,728(37)$ & & $327(28)$ & $1,627(28)$ & \\
\hline \multicolumn{10}{|l|}{ Age at diagnosis (years) } \\
\hline$<50$ & $339(24)$ & $1,667(23)$ & N.A. & $90(6)$ & $330(5)$ & N.A. & $6(1)$ & $20(0)$ & N.A. \\
\hline 50 to 59 & $286(20)$ & $1,430(20)$ & & $226(15)$ & $1,117(15)$ & & $71(6)$ & $351(6)$ & \\
\hline 60 to 69 & $273(19)$ & $1,365(19)$ & & $401(27)$ & $2,001(27)$ & & $280(24)$ & $1,400(24)$ & \\
\hline 70 to 79 & $318(22)$ & $1,588(22)$ & & $496(33)$ & $2,472(34)$ & & $532(45)$ & $2,660(45)$ & \\
\hline$\geq 80$ & $219(15)$ & $1,056(15)$ & & $298(20)$ & $1,371(19)$ & & $295(25)$ & $1,418(24)$ & \\
\hline Male & $0(0)$ & $0(0)$ & N.A. & $871(58)$ & $4,231(58)$ & N.A. & $1,184(100)$ & $5,849(100)$ & N.A. \\
\hline \multicolumn{10}{|l|}{ Exposure period (years): } \\
\hline Mean (sd) & $3.9(2.3)$ & $3.9(2.3)$ & N.A. & $2.8(1.6)$ & $2.9(1.7)$ & N.A. & $3.8(2.2)$ & $3.7(2.2)$ & N.A. \\
\hline Range & 1.0 to 12.8 & 1.0 to 12.8 & N.A. & 1.0 to 10.5 & 1.0 to 10.5 & N.A. & 1.0 to 11.9 & 1.0 to 11.9 & \\
\hline Stage:1 & $72(11)$ & $1,471(43)$ & $<0.001$ & $61(6)$ & $1,044(18)$ & $<0.001$ & & & \\
\hline 2 & $402(61)$ & $1,676(49)$ & & $275(25)$ & $2,496(44)$ & & & & \\
\hline 3 & $116(18)$ & $220(6)$ & & $558(51)$ & 1,981 (35) & & & & \\
\hline 4 & $64(10)$ & $40(1)$ & & 203 (19) & $195(3)$ & & & & \\
\hline Missing & 781 & 3,699 & & 414 & 1,575 & & & & \\
\hline Grade:Well & $54(6)$ & 751 (19) & & $81(7)$ & $562(9)$ & $<0.001$ & $34(4)$ & $538(13)$ & $<0.001$ \\
\hline Moderate & $377(41)$ & $1,900(49)$ & & $889(75)$ & 4,894 (78) & & $217(28)$ & $2,067(48)$ & \\
\hline Poor & $492(53)$ & $1,217(31)$ & & $208(17)$ & $818(13)$ & & $516(67)$ & $1,683(39)$ & \\
\hline Missing & 512 & 3,280 & & 333 & 1017 & & 417 & 1561 & \\
\hline \multicolumn{10}{|l|}{$\begin{array}{l}\text { Treatment within six months } \\
\text { of diagnosis }\end{array}$} \\
\hline Chemotherapy & $573(40)$ & $1,611(23)$ & $<0.001$ & $651(43)$ & $1,916(26)$ & $<0.001$ & $49(4)$ & $137(2)$ & $<0.001$ \\
\hline Radiotherapy & 700 (49) & $3,355(47)$ & 0.22 & $302(20)$ & $1,066(15)$ & $<0.001$ & $246(21)$ & $1,276(22)$ & 0.48 \\
\hline Surgery & $1,087(76)$ & $5,982(84)$ & $<0.001$ & $1,214(80)$ & 65,15 (89) & $<0.001$ & & & \\
\hline Tamoxifen therapy & 709 (49) & $4,610(65)$ & $<0.001$ & & & & & & \\
\hline Androgen therapy & & & & & & & $976(82)$ & $3,450(59)$ & $<0.001$ \\
\hline Radical prostatectomy ${ }^{a}$ & & & & & & & $20(2)$ & $331(8)$ & $<0.001$ \\
\hline Smoking prior to diagnosis & & & 0.03 & & & 0.01 & & & $<0.001$ \\
\hline Non-smoker & $710(60)$ & $3,827(64)$ & & $640(51)$ & $3,138(52)$ & & $453(47)$ & $2,541(52)$ & \\
\hline Ex-smoker & $226(19)$ & $1,072(18)$ & & $365(29)$ & $1,885(31)$ & & $331(34)$ & $1,660(34)$ & \\
\hline Current smoker & $249(21)$ & $1,094(18)$ & & $249(20)$ & $983(16)$ & & $185(19)$ & $674(14)$ & \\
\hline Missing & 250 & 1,113 & & 257 & 1285 & & 215 & 974 & \\
\hline BMI prior to diagnosis & & & 0.01 & & & 0.66 & & & 0.05 \\
\hline Mean (sd) & $26.6(5.5)$ & $26.2(5.1)$ & & $26.5(4.7)$ & $26.6(4.8)$ & & $26.4(4.0)$ & $26.1(3.7)$ & \\
\hline Missing & 386 & 1,633 & & 412 & 1,810 & & 302 & 1,362 & \\
\hline Alcohol prior to diagnosis & & & 0.58 & & & 0.47 & & & 0.37 \\
\hline Alcohol consumer & $831(80)$ & $4,333(81)$ & & $950(87)$ & $4,585(86)$ & & 791 (89) & $4,047(90)$ & \\
\hline Missing & 401 & 1,779 & & 420 & 1,977 & & 298 & 1366 & \\
\hline
\end{tabular}


Table 1 Characteristics of cancer patients who die from cancer (cases) compared with controls, by cancer site (Continued)

\begin{tabular}{|c|c|c|c|c|c|c|c|c|c|}
\hline \multicolumn{10}{|c|}{$\begin{array}{l}\text { Comorbidity (prior to diagnosis } \\
\text { or during follow-up time) }\end{array}$} \\
\hline Cerebrovascular & $89(6)$ & $383(5)$ & 0.53 & $117(8)$ & $493(7)$ & 0.19 & $124(10)$ & $509(9)$ & 0.07 \\
\hline C.P.D. & $264(18)$ & $1,247(18)$ & 0.60 & $236(16)$ & $1295(18)$ & 0.06 & $229(19)$ & $1,110(19)$ & 0.80 \\
\hline C.H.D. & $60(4)$ & $249(4)$ & 0.68 & $91(6)$ & $367(5)$ & 0.13 & $108(9)$ & $395(7)$ & 0.01 \\
\hline Diabetes & $126(9)$ & $468(7)$ & 0.02 & $192(13)$ & $866(12)$ & 0.21 & $141(12)$ & $619(11)$ & 0.17 \\
\hline Myocardial infarction & $26(2)$ & $148(2)$ & 0.74 & $104(7)$ & $454(6)$ & 0.32 & $130(11)$ & $522(9)$ & 0.03 \\
\hline Peptic ulcer disease & $39(3)$ & $184(3)$ & 0.79 & $111(7)$ & $477(7)$ & 0.19 & $77(7)$ & $440(8)$ & 0.18 \\
\hline P.V.D. & $51(4)$ & $165(2)$ & 0.003 & $64(4)$ & $331(5)$ & 0.70 & $113(10)$ & $396(7)$ & 0.001 \\
\hline Renal disease & $78(5)$ & $382(5)$ & 0.92 & $107(7)$ & $478(7)$ & 0.45 & $138(12)$ & $509(9)$ & 0.001 \\
\hline Rheumatological & $76(5)$ & $330(5)$ & 0.35 & $55(4)$ & $272(4)$ & 0.82 & $37(3)$ & $287(5)$ & 0.01 \\
\hline
\end{tabular}

${ }^{a}$ Excludes cancer registries without available data. C.P.D., chronic pulmonary disease; C.H.D., congestive heart disease; N.A., not applicable; P.V.D., peripheral vascular disease; sd, standard deviation.

approximately $80 \%$ power to detect, at the $5 \%$ significance level, an OR for cancer-specific mortality in ACEI users of 1.25 and for ARB users of 1.35 in each of the cancer cohorts. Statistical analyses were conducted in STATA 11 (StataCorp, College Station, Texas).

\section{Results}

\section{Patient cohorts}

Figure 1 shows the selection of the final cohorts. The average follow-up (in those not dying) was six years (range one to thirteen years) in each of the three cohorts. The crude rate of cancer-specific death was 28.0 per 1,000 person years in the breast cancer cohort (based upon 1,440 breast cancer-specific deaths in 51,507 person years), 78.5 per 1,000 person years in the colorectal cancer cohort (based upon 1,528 colorectal cancer-specific deaths in 19,462 person years), and 41.2 per 1,000 person years in the prostate cancer cohort (based upon 1,194 prostate cancerspecific deaths in 28,970 person years). These cohorts were converted to case-control data for the cancer-specific mortality analysis with 1,435 breast, 1,511 colorectal and 1,184 prostate cases (cancer-specific deaths) and 7,106 breast, 7,291 colorectal and 5,849 prostate risk-set controls.

\section{Patient characteristics}

Table 1 shows characteristics of cancer-specific deaths (cases) and controls. The average time to death and, hence, the end of the exposure period was 3.9, 2.8 and 3.8 years for breast, colorectal and prostate cancer, respectively. Cancer-specific deaths (cases) had higher stage, higher histological grade, more chemotherapy and less surgery. A higher proportion of cases had radiotherapy for colorectal but not breast or prostate cancer. Hormone therapy was less frequent in breast cancer cases but more frequent in prostate cancer cases. Smoking rates were slightly higher in cases. Rates of comorbidities, alcohol consumption and BMI levels prior to diagnosis were generally similar between cases and controls (Table 1).

\section{ACEI/ARB use and mortality in breast cancer patients}

In breast cancer patients (Table 2), the ACEI prescriptions after diagnosis were similar in those dying from cancer (cases) compared with controls (16.9\% versus $16.4 \%$, respectively; $\mathrm{OR}=1.04,95 \% \mathrm{CI} 0.88,1.22)$. Adjustment for potential confounders did not alter this OR markedly $(\mathrm{OR}=1.06,95 \%$ CI $0.89,1.27)$. Similarly, no associations were observed between cancer-specific mortality and ARB use (adjusted OR $=1.06,95 \%$ CI 0.84, 1.35). These findings were little altered after adjustment for stage and were fairly consistent across sensitivity analyses (shown in Table 3). No associations were observed with pre-diagnostic use (Table 3). Finally, analyses of post-diagnostic ACEI (adjusted OR $=1.04,95 \%$ CI 0.92, 1.19) and ARB (adjusted $\mathrm{OR}=0.96,95 \%$ CI $0.81,1.14$ ) use and all-cause mortality showed little evidence of associations (Table 4).

\section{ACEI/ARB use and mortality in colorectal cancer patients}

In colorectal cancer patients (Table 2), there was some evidence of a reduction in cancer-specific mortality in patients prescribed an ACEI (OR $=0.79,95 \%$ CI 0.69, 0.92) which was little altered after adjustment for confounders (adjusted OR $=0.78,95 \%$ CI 0.66, 0.92), but a clear dose response association was not apparent as there was little evidence of protective effects in those using more than 365 DDDs of ACEI (adjusted OR $=0.8495 \%$ CI 0.68, 1.03). There was little evidence of an association between ARBs and cancer-specific mortality (adjusted OR $=0.82$ 95\% CI $0.64,1.07)$. Additional adjustment for stage little altered these estimates. Sensitivity analyses (Table 5) produced fairly consistent results for ARBs. Any protective association for ACEIs were attenuated when prescriptions in the year prior to death were removed (adjusted OR $=0.83$ 95\% 
Table 2 Association between post-diagnostic exposure to ACEls and ARBS and cancer specific death, by cancer site

\begin{tabular}{|c|c|c|c|c|c|c|c|c|c|c|}
\hline \multirow{2}{*}{$\begin{array}{l}\text { Post-diagnostic } \\
\text { medication usage }\end{array}$} & \multicolumn{6}{|c|}{ All cancer patients } & \multicolumn{4}{|c|}{ Cancer patients with available stage/grade ${ }^{c}$} \\
\hline & $\begin{array}{c}\text { Cancer specific } \\
\text { deaths number (\%) }\end{array}$ & $\begin{array}{c}\text { Controls } \\
\text { number (\%) }\end{array}$ & $\begin{array}{l}\text { Unadjusted } \\
\text { OR (95\% Cl) }\end{array}$ & $P\left(\right.$ trend $\left.^{\mathrm{a}}\right)$ & $\begin{array}{l}\text { Adjusted }^{\text {b }} \\
\text { OR }(95 \% \mathrm{Cl})\end{array}$ & $P\left(\right.$ trend $\left.^{\mathrm{a}}\right)$ & $\begin{array}{l}\text { Unadjusted } \\
\text { OR }(95 \% \mathrm{Cl})\end{array}$ & $P\left(\right.$ trend $\left.^{\mathrm{a}}\right)$ & $\begin{array}{l}\text { Fully adjusted } \\
\text { OR }(95 \% \mathrm{Cl})^{d}\end{array}$ & $P\left(\right.$ trend $\left.^{\mathrm{a}}\right)$ \\
\hline & & & Breast cancer & & & & & & & \\
\hline \multicolumn{11}{|l|}{ ACEI } \\
\hline 0 (non-user) & $1,192(83.1)$ & $5,938(83.6)$ & 1.00 (ref. cat.) & & 1.00 (ref. cat.) & & 1.00 (ref. cat.) & & 1.00 (ref. cat.) & \\
\hline \multirow[t]{2}{*}{$\geq 1$ DDD (user) } & $243(16.9)$ & $1,168(16.4)$ & $1.04(0.88,1.22)$ & 0.65 & $1.06(0.89,1.27)$ & 0.52 & $0.96(0.76,1.21)$ & 0.72 & $0.83(0.63,1.09)$ & 0.18 \\
\hline & & & & $(0.50)$ & & $(0.32)$ & & (0.88) & & $(0.47)$ \\
\hline 1to 365 DDDs & $92(6.4)$ & $469(6.6)$ & $0.97(0.77,1.23)$ & 0.80 & $0.96(0.75,1.24)$ & 0.78 & $0.88(0.61,1.27)$ & 0.49 & $0.65(0.43,0.99)$ & 0.04 \\
\hline$\geq 365$ DDDs & $151(10.5)$ & $699(9.8)$ & $1.08(0.89,1.32)$ & 0.42 & $1.14(0.92,1.41)$ & 0.25 & $1.01(0.76,1.33)$ & 0.96 & $0.96(0.69,1.34)$ & 0.82 \\
\hline \multicolumn{11}{|l|}{ ARB } \\
\hline 0 (non-user) & 1,333 (92.9) & 6,602 (92.9) & 1.00 (ref. cat.) & & 1.00 (ref. cat.) & & 1.00 (ref. cat.) & & 1.00 (ref. cat.) & \\
\hline \multirow[t]{2}{*}{$\geq 1$ DDD (user) } & $102(7.1)$ & $504(7.1)$ & $1.00(0.80,1.26)$ & 0.98 & $1.06(0.84,1.35)$ & 0.62 & $1.03(0.84,1.25)$ & 0.80 & $0.94(0.65,1.37)$ & 0.75 \\
\hline & & & & $(0.99)$ & & $(0.57)$ & & $(0.69)$ & & $(0.85)$ \\
\hline 1 to 365 DDDs & $33(2.3)$ & $160(2.3)$ & $1.02(0.70,1.51)$ & 0.87 & $1.01(0.68,1.51)$ & 0.96 & $1.04(0.58,1.87)$ & 0.90 & $0.84(0.44,1.61)$ & 0.59 \\
\hline \multirow[t]{2}{*}{$\geq 365$ DDDs } & $69(4.8)$ & $344(4.8)$ & $0.99(0.75,1.30)$ & 0.95 & $1.09(0.82,1.45)$ & 0.55 & $1.08(0.73,1.60)$ & 0.70 & $0.99(0.64,1.52)$ & 0.95 \\
\hline & & & Colorectal cance & & & & & & & \\
\hline \multicolumn{11}{|l|}{ ACEI } \\
\hline 0 (non-user) & $1,231(81.5)$ & $5,660(77.6)$ & 1.00 (ref. cat.) & & 1.00 (ref. cat.) & & 1.00 (ref. cat.) & & 1.00 (ref. cat.) & \\
\hline \multirow[t]{2}{*}{$\geq 1$ DDD (user) } & $280(18.5)$ & $1,631(22.4)$ & $0.79(0.69,0.92)$ & 0.002 & $0.78(0.66,0.92)$ & 0.003 & $0.75(0.63,0.89)$ & 0.001 & $0.76(0.62,0.93)$ & 0.01 \\
\hline & & & & $(0.01)$ & & $(0.02)$ & & $(0.005)$ & & $(0.02)$ \\
\hline 1 to 365 DDDs & $113(7.5)$ & $729(10.0)$ & $0.72(0.59,0.89)$ & 0.002 & $0.71(0.57,0.89)$ & 0.03 & $0.69(0.54,0.90)$ & 0.005 & $0.71(0.53,0.94)$ & 0.02 \\
\hline$\geq 365$ DDDs & $167(11.1)$ & $902(12.4)$ & $0.85(0.71,1.02)$ & 0.09 & $0.84(0.68,1.03)$ & 0.10 & $0.80(0.64,0.98)$ & 0.03 & $0.80(0.63,1.02)$ & 0.07 \\
\hline \multicolumn{11}{|l|}{ ARB } \\
\hline 0 (non-user) & $1,428(94.5)$ & 6,811 (93.4) & 1.00 (ref. cat.) & & 1.00 (ref. cat.) & & 1.00 (ref. cat.) & & 1.00 (ref. cat.) & \\
\hline \multirow[t]{2}{*}{$\geq 1$ DDD (user) } & $83(5.5)$ & $480(6.6)$ & $0.84(0.66,1.07)$ & 0.15 & $0.82(0.64,1.07)$ & 0.14 & $0.91(0.76,1.08)$ & 0.29 & $0.80(0.59,1.09)$ & 0.16 \\
\hline & & & & $(0.41)$ & & $(0.94)$ & & $(0.26)$ & & $(0.66)$ \\
\hline 1 to 365 DDDs & $29(1.9)$ & $221(3.0)$ & $0.64(0.43,0.95)$ & 0.03 & $0.63(0.42,0.94)$ & 0.03 & $0.51(0.31,0.83)$ & 0.01 & $0.61(0.37,1.03)$ & 0.06 \\
\hline \multirow[t]{2}{*}{$\geq 365$ DDDs } & $54(3.6)$ & 259 (3.6) & $1.00(0.74,1.36)$ & 0.98 & $1.00(0.72,1.37)$ & 0.98 & $0.98(0.69,1.38)$ & 0.89 & $0.93(0.64,1.36)$ & 0.72 \\
\hline & & & Prostate cancer & & & & & & & \\
\hline \multicolumn{11}{|l|}{ ACEI } \\
\hline 0 (non-user) & 848 (71.6) & 4,145 (70.9) & 1.00 (ref. cat.) & & 1.00 (ref. cat.) & & 1.00 (ref. cat.) & & 1.00 (ref. cat.) & \\
\hline \multirow[t]{2}{*}{$\geq 1$ DDD (user) } & $336(28.4)$ & $1,704(29.1)$ & $0.96(0.83,1.10)$ & 0.55 & $0.78(0.66,0.92)$ & 0.003 & $0.98(0.82,1.17)$ & 0.80 & $0.82(0.67,1.02)$ & 0.07 \\
\hline & & & & $(0.47)$ & & $(0.003)$ & & (0.85) & & $(0.11)$ \\
\hline
\end{tabular}


Table 2 Association between post-diagnostic exposure to ACEls and ARBS and cancer specific death, by cancer site (Continued)

\begin{tabular}{|c|c|c|c|c|c|c|c|c|c|c|}
\hline 1 to 365 DDDs & $118(10.0)$ & $572(9.8)$ & $1.00(0.81,1.24)$ & 0.99 & $0.83(0.66,1.04)$ & 0.11 & $0.96(0.74,1.26)$ & 0.79 & $0.80(0.59,1.07)$ & 0.14 \\
\hline$\geq 365$ DDDs & $218(18.4)$ & 1,132 (19.4) & $0.94(0.79,1.11)$ & 0.44 & $0.75(0.62,0.91)$ & 0.003 & $0.98(0.80,1.21)$ & 0.88 & $0.84(0.66,1.07)$ & 0.16 \\
\hline \multicolumn{11}{|l|}{ ARB } \\
\hline 0 (non-user) & $1,103(93.2)$ & $5,384(92.1)$ & 1.00 (ref. cat.) & & 1.00 (ref. cat.) & & 1.00 (ref. cat.) & & 1.00 (ref. cat.) & \\
\hline \multirow[t]{2}{*}{$\geq 1$ DDD (user) } & $81(6.8)$ & $465(8.0)$ & $0.86(0.67,1.10)$ & 0.22 & $0.79(0.61,1.03)$ & 0.08 & $0.93(0.70,1.23)$ & 0.62 & $0.82(0.61,1.11)$ & 0.21 \\
\hline & & & & $(0.16)$ & & $(0.06)$ & & $(0.75)$ & & $(0.26)$ \\
\hline 1 to 365 DDDs & $30(2.5)$ & $153(2.6)$ & $0.97(0.65,1.44)$ & 0.88 & $0.88(0.58,1.33)$ & 0.55 & $0.84(0.52,1.36)$ & 0.48 & $0.77(0.46,1.29)$ & 0.33 \\
\hline$\geq 365$ DDDs & $51(4.3)$ & $312(5.3)$ & $0.80(0.59,1.09)$ & 0.15 & $0.74(0.54,1.02)$ & 0.07 & $0.98(0.70,1.36)$ & 0.90 & $0.84(0.59,1.21)$ & 0.36 \\
\hline
\end{tabular}

a Tests for trend from conditional logistic regression model based upon categories of DDDs $\left(0,1\right.$ to $365, \geq 365$ DDDs coded as $0,1,2$, respectively). ${ }^{b} A l l$ models include surgery (within six months of diagnosis), chemotherapy (within six months), radiotherapy (within six months), low dose aspirin (during exposure period), statins (during exposure period), comorbidities (pre-diagnosis or during exposure period, including myocardial infarction, cerebrovascular disease, congestive heart disease, chronic pulmonary disease, peripheral vascular disease, renal disease, peptic ulcer disease and diabetes), and smoking (pre-diagnosis, with missing included as a category). The breast cancer model additionally includes tamoxifen (within six months), aromatase inhibitors (within six months), hormone replacement therapy (pre-diagnosis). The prostate cancer model additionally includes androgen therapy (within six months). 'Analysis includes 1,093 cases and 5,231 controls in colorectal cancer patients with available stage, 648 cases and 3,193 controls in breast cancer patients with available stage and 766 cases and 3,777 controls in prostate cancer patients with available grade. ${ }^{\mathrm{d} A d d i t i o n a l l y}$ adjusted for stage in colorectal cancer patients and breast cancer patients and adjusted for grade in prostate cancer patients. ACEls, angiotensin-converting enzyme inhibitors; ARBs, angiotensin II receptor blockers; Cl, confidence interval; DDDs, daily defined doses; OR, odds ratio. 


\begin{tabular}{|c|c|c|c|c|}
\hline & & Breast cancer & Colorectal cancer & Prostate cancer \\
\hline \multirow{2}{*}{\multicolumn{2}{|c|}{$\begin{array}{l}\text { Patients diagnosed } 1998-2007 \text { with CPRD } \\
\text { coverage and no previous cancer. }\end{array}$}} & 11,863 breast cancer patients & 7,081 colorectal cancer patients & 8,128 prostate cancer patients \\
\hline & & $\sqrt{3}$ & $\sqrt{2}$ & $\sqrt{3}$ \\
\hline \multirow{2}{*}{\multicolumn{2}{|c|}{$\begin{array}{l}\text { After excluding patients where cancer diagnosis } \\
\text { preceded CPRD research quality records." }\end{array}$}} & 10,969 breast cancer patients & 6,666 colorectal cancer patients & 7,495 prostate cancer patients \\
\hline & & $\sqrt{2}$ & $\sqrt{2}$ & $\sqrt{3}$ \\
\hline \multirow{2}{*}{\multicolumn{2}{|c|}{$\begin{array}{l}\text { After excluding patients where death } \\
\text { registration data unavailable. }\end{array}$}} & 10,853 breast cancer patients & 6,540 colorectal cancer patients & 7,388 prostate cancer patients \\
\hline & & $\sqrt{2}$ & $\sqrt{2}$ & $\sqrt{2}$ \\
\hline \multirow{2}{*}{\multicolumn{2}{|c|}{$\begin{array}{l}\text { After excluding patients with }<1 \text { year follow- } \\
\text { up. }\end{array}$}} & 9,971 breast cancer patients & 4,762 colorectal cancer patients & 6,513 prostate cancer patients \\
\hline & & $\sqrt{2}$ & $\sqrt{2}$ & $\sqrt{2}$ \\
\hline \multirow{2}{*}{\multicolumn{2}{|c|}{$\begin{array}{l}\text { After excluding patients where hormone therapy } \\
\text { preceded cancer diagnosis by } 8 \text { weeks. }\end{array}$}} & 9,814 breast cancer patients & 4,762 colorectal cancer patients & 6,339 prostate cancer patients \\
\hline & & 马 & そ & Љ \\
\hline \multirow{4}{*}{\multicolumn{2}{|c|}{ Final cohort for analysis. }} & 9,814 breast cancer patients & 4,762 colorectal cancer patients & 6,339 prostate cancer patients \\
\hline & & 1,440 cancer specific deaths & 1,528 cancer specific deaths & 1,194 cancer specific deaths \\
\hline & & 2,271 all-cause deaths & 2,134 all-cause deaths & 2,213 all-cause deaths \\
\hline & & $\checkmark$ & 亿 & \ \\
\hline \multirow[t]{2}{*}{$\begin{array}{l}\text { Data for analysis after } \\
\text { conversion to case- } \\
\text { control data. }\end{array}$} & $\begin{array}{l}\text { Cancer-specific } \\
\text { mortality analysis }\end{array}$ & $\begin{array}{l}1,435 \text { cancer-specific deaths (cases) } \\
7,106 \text { matched controls } \\
(5 \text { cases had no available match) }\end{array}$ & $\begin{array}{l}1,511 \text { cancer-specific deaths } \\
7,291 \text { matched controls } \\
\text { (17 cases had no available match) }\end{array}$ & $\begin{array}{l}\text { 1,184 cancer-specific deaths } \\
5,849 \text { matched controls } \\
\text { (10 cases had no available match) }\end{array}$ \\
\hline & $\begin{array}{l}\text { All-cause mortality } \\
\text { analysis }\end{array}$ & $\begin{array}{l}2,258 \text { all-cause deaths } \\
11,154 \text { matched controls } \\
\text { (13 cases had no available match) }\end{array}$ & $\begin{array}{l}2,101 \text { all-cause deaths } \\
10,107 \text { matched controls } \\
\text { ( } 33 \text { cases had no available match) }\end{array}$ & $\begin{array}{l}2,197 \text { all-cause deaths } \\
10,838 \text { matched controls } \\
\text { (16 cases no available match) }\end{array}$ \\
\hline \multicolumn{5}{|c|}{ 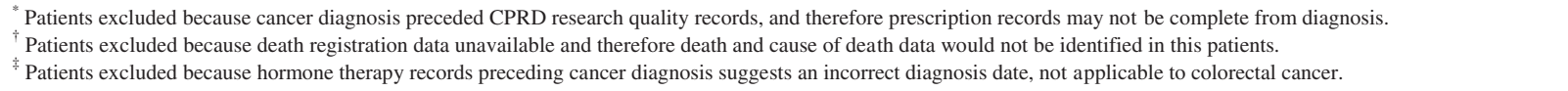 } \\
\hline \multicolumn{5}{|c|}{ Figure 1 Flow chart of selection of patients for inclusion in analysis, by cancer site. } \\
\hline
\end{tabular}

CI $0.67,1.03$ ) or when the analysis was restricted to users of any antihypertensive medication in the year prior to cancer diagnosis (adjusted OR $=0.83$ 95\% CI 0.65, 1.07). In early stage colorectal cancer a more marked reduction in cancer specific mortality was observed with ACEI use (adjusted OR $=0.54$ 95\% CI 0.38, 0.77) compared with later stage disease (adjusted $\mathrm{OR}=0.86,95 \% \mathrm{CI}$ $0.68,1.10$; $P$ for interaction $=0.03$ ). In general, any protective associations were less marked when using the time varying covariate approach but other sensitivity analyses for ACEIs were similar to the main finding. No associations were observed between cancer-specific mortality and pre-diagnostic ACEI and ARB use (Table 3). Finally, there was little evidence of associations with all-cause mortality and ACEI (adjusted OR $=0.90,95 \% \mathrm{CI}$ $0.79,1.02$ ) or $\mathrm{ARB}$ (adjusted $\mathrm{OR}=0.94,95 \% \mathrm{CI} 0.78,1.13$ ) use after cancer diagnosis (Table 4).

\section{ACEI/ARB use and mortality in prostate cancer patients}

In prostate cancer patients (Table 2), there was no evidence of an increase in cancer-specific mortality in ACEI users (adjusted $\mathrm{OR}=0.78,95 \% \mathrm{CI} 0.66,0.92$ ) or $\mathrm{ARB}$ users (adjusted OR $=0.79,95 \%$ CI 0.61, 1.03). Additionally, adjusting for grade little altered the point estimates although the protective association with ACEIs was no longer significant (fully adjusted OR $=0.8295 \%$ CI $0.67,1.02$ ). The associations for ARB use were fairly consistent across sensitivity analyses (Table 6). The protective association with ACEIs was more marked when restricting the analysis to antihypertensive users during the exposure period (adjusted $\mathrm{OR}=0.7895 \% \mathrm{CI} 0.63,0.96$ ) and when adjusting for other antihypertensive use (adjusted OR $=0.74$ 95\% CI $0.60,0.92)$, but were attenuated in most other sensitivity analyses particularly when prescriptions in the year prior to death were removed (adjusted OR $=0.88$ 95\% CI 0.70, 1.09) and in time-varying covariate analyses (adjusted hazard ratio $(\mathrm{HR})=0.9095 \% \mathrm{CI} 0.76,1.07)$. There was little evidence of a reduction in all-cause mortality in ACEI users (adjusted OR $=0.91,95 \%$ CI $0.82,1.03$ ) or $\mathrm{ARB}$ users (adjusted OR $=0.85$, 95\% CI 0.72, 1.01) after cancer diagnosis (Table 4).

\section{Discussion}

Overall, we found no evidence of increased risks of cancer-specific or all-cause mortality in breast, colorectal or prostate cancer patients using ACEIs or ARBs after cancer diagnosis. There was some evidence of reductions in the risk of cancer-specific mortality in colorectal and 
Table 3 Sensitivity analyses for association between ACEls and ARBS and cancer specific mortality in breast cancer patients

\begin{tabular}{|c|c|c|c|c|c|c|}
\hline & \multirow{2}{*}{$\begin{array}{l}\text { Cancer specific } \\
\text { deaths number }\end{array}$} & \multirow{2}{*}{$\begin{array}{l}\text { Controls } \\
\text { number }\end{array}$} & \multirow{2}{*}{$\begin{array}{c}\text { OR }(95 \% \mathrm{Cl}) \\
\begin{array}{c}\text { ACEI user versus } \\
\text { non user }\end{array}\end{array}$} & \multirow[t]{2}{*}{$P$} & \multirow{2}{*}{$\begin{array}{c}\text { OR }(95 \% \mathrm{Cl}) \\
\begin{array}{c}\text { ARB user versus } \\
\text { non user }\end{array}\end{array}$} & \multirow[t]{2}{*}{$P$} \\
\hline & & & & & & \\
\hline \multicolumn{7}{|l|}{ Breast cancer } \\
\hline Main analysis: diagnosis to six months prior to death ${ }^{\mathrm{a}}$ & 648 & 3,193 & $0.83(0.63,1.09)$ & 0.18 & $0.94(0.65,1.37)$ & 0.75 \\
\hline Diagnosis to 1 year prior to death ${ }^{\mathrm{b}}$ & 583 & 2,875 & $0.87(0.65,1.16)$ & 0.33 & $0.87(0.58,1.30)$ & 0.49 \\
\hline $\begin{array}{l}\text { Restricted to users of any antihypertensive medication }{ }^{c} \text { prior } \\
\text { to cancer diagnosis }{ }^{d}\end{array}$ & 212 & 994 & $0.87(0.60,1.25)$ & 0.44 & $1.36(0.85,2.16)$ & 0.20 \\
\hline $\begin{array}{l}\text { Comparison group restricted to users of any antihypertensive } \\
\text { in exposure period }\end{array}$ & 361 & 1,624 & $0.79(0.59,1.05)$ & 0.10 & $0.92(0.63,1.34)$ & 0.67 \\
\hline Additionally adjusting for other antihypertensives ${ }^{\mathrm{e}}$ & 648 & 3,193 & $0.79(0.60,1.06)$ & 0.12 & $0.90(0.61,1.33)$ & 0.60 \\
\hline Additionally adjusting for hormone therapy any time after diagnosis ${ }^{f}$ & 648 & 3,193 & $0.80(0.60,1.05)$ & 0.11 & $0.98(0.66,1.44)$ & 0.91 \\
\hline$\geq 730$ DDDs versus 0 DDDs (non-user) & 648 & 3,193 & $1.06(0.74,1.52)$ & 0.75 & $0.99(0.64,1.52)$ & 0.95 \\
\hline $\begin{array}{l}\text { Restricted to cancer registries with stage data available for over } \\
85 \% \text { of patients }\end{array}$ & 487 & 1,911 & $0.84(0.59,1.18)$ & 0.31 & $1.33(0.81,2.17)$ & 0.25 \\
\hline Stage 1 and $2^{g}$ & 469 & 2,313 & $0.95(0.68,1.32)$ & 0.75 & $1.14(0.73,1.81)$ & 0.56 \\
\hline Stage 3 and $4^{9}$ & 161 & 531 & $0.83(0.46,1.51)$ & 0.54 & $2.37(1.05,5.35)$ & 0.04 \\
\hline Pre-diagnostic use ${ }^{\mathrm{h}}$ & 695 & 3,413 & $0.86(0.61,1.20)$ & 0.37 & $1.06(0.65,1.72)$ & 0.82 \\
\hline Time varying covariate analysis ${ }^{i}$ & 656 & 4,164 & & & & \\
\hline$\geq 1$ DDDs versus 0 DDDs (non-user) & & & $0.97(0.77,1.21)$ & 0.76 & $1.20(0.89,1.62)$ & 0.23 \\
\hline 1to 365 DDDs versus 0 DDDs (non-user) & & & $0.90(0.65,1.25)$ & 0.52 & $0.94(0.55,1.60)$ & 0.81 \\
\hline$\geq 365$ DDDs versus 0 DDDs (non-user) & & & $1.01(0.77,1.32)$ & 0.93 & $1.35(0.96,1.91)$ & 0.09 \\
\hline
\end{tabular}

${ }^{a}$ Except where otherwise stated analysis investigates medications from diagnosis to six months prior to death/index date and models include surgery (within six months of diagnosis), chemotherapy (within six months), radiotherapy (within six months), low dose aspirin (during exposure period), statins (during exposure period), comorbidities (pre-diagnosis or during exposure period, including myocardial infarction, cerebrovascular disease, congestive heart disease, chronic pulmonary disease, peripheral vascular disease, renal disease, peptic ulcer disease and diabetes), and smoking (pre-diagnosis, with missing included as a category), tamoxifen (within six months), aromatase inhibitors (within six months), hormone replacement therapy (pre-diagnosis) and stage. ${ }^{\mathrm{b}}$ Restricted to individuals surviving more than 1.5 years. ${ }^{\mathrm{C}}$ Antihypertensive medications include beta-blockers, diuretics, vasodilator antihypertensive drugs, centrally acting antihypertensive drugs, alpha-adrenoceptor blocking drugs, ACEIs, ARBs, renin inhibitors, and calcium channel blockers. ${ }^{\mathrm{d}}$ Restricted to individuals with $>1$ year of records prior to cancer diagnosis and pre-diagnostic use considered antihypertensive use in that year, excluding deaths in the year after cancer diagnosis. ${ }^{~}$ Models include all variables in and additionally include calcium channel blockers, diuretics and beta-blockers. ${ }^{f}$ Models include all variables in ${ }^{a}$ but tamoxifen and aromatase inhibitors were determined at any time after diagnosis in the exposure period. ${ }^{9}$ Stratified analyses were conducted after re-matching controls to cases within strata; due to non-availability of matches overall numbers in subgroups may not be identical to numbers presented in Table 1. "hestricted to individuals with $>1$ year of records prior to cancer diagnosis and pre-diagnostic use considered ACEl/ARB use in that year, not excluding deaths in the year after cancer diagnosis. 'Reported estimates are adjusted hazard ratios and $95 \%$ Cls, model includes age at diagnosis, year of diagnosis, surgery (within six months of diagnosis), chemotherapy (within six months), radiotherapy (within six months), statins (during exposure period, as a time varying covariate), comorbidities (pre-diagnosis including, myocardial infarction, cerebrovascular disease, congestive heart disease, chronic pulmonary disease, peripheral vascular disease, renal disease, peptic ulcer disease and diabetes) and stage. ACEls, angiotensin-converting enzyme inhibitors; ARBs, angiotensin II receptor blockers; $\mathrm{Cl}$, confidence interval; DDDs, daily defined doses; OR, odds ratio.

prostate cancer patients using ACEIs, but any protective effects were weak in magnitude, were inconsistent across sensitivity analyses and were not a priori stated and, therefore, are difficult to interpret.

\section{Strengths and weaknesses}

The main strengths of our study were the large size (including over 20,000 cancer patients and 4,000 cancerspecific deaths) and the long duration of follow-up (up to 13 years) which provided the ability to detect relatively weak effects and to report narrow CIs which rule out relatively small increases in risk. Our study used GP prescribed drug information which captures almost all ACEI and ARB use (which are only available by prescription in the UK), eliminates the potential for recall bias incurred by self-report and allows detailed temporal associations to be explored. Consequently, our data reflect GP prescriptions rather than drug consumption but analyses of multiple prescriptions generally found similar results, suggesting compliance may not impact our results greatly. As with all observational studies we cannot exclude residual or unknown confounding. We had robust data on surgery, radiotherapy, chemotherapy, hormone therapy, comorbidities and importantly stage for colorectal and breast and Gleason score for prostate cancer, but we had limited data on smoking and alcohol intake and no information on socioeconomic status.

\section{Comparison with previous studies}

In prostate and colorectal cancer our study is the first epidemiological study to investigate ARBs separately and cancer progression and only three studies have investigated 
Table 4 Association between post-diagnostic exposure to ACEls and ARBS and all-cause mortality, by cancer site

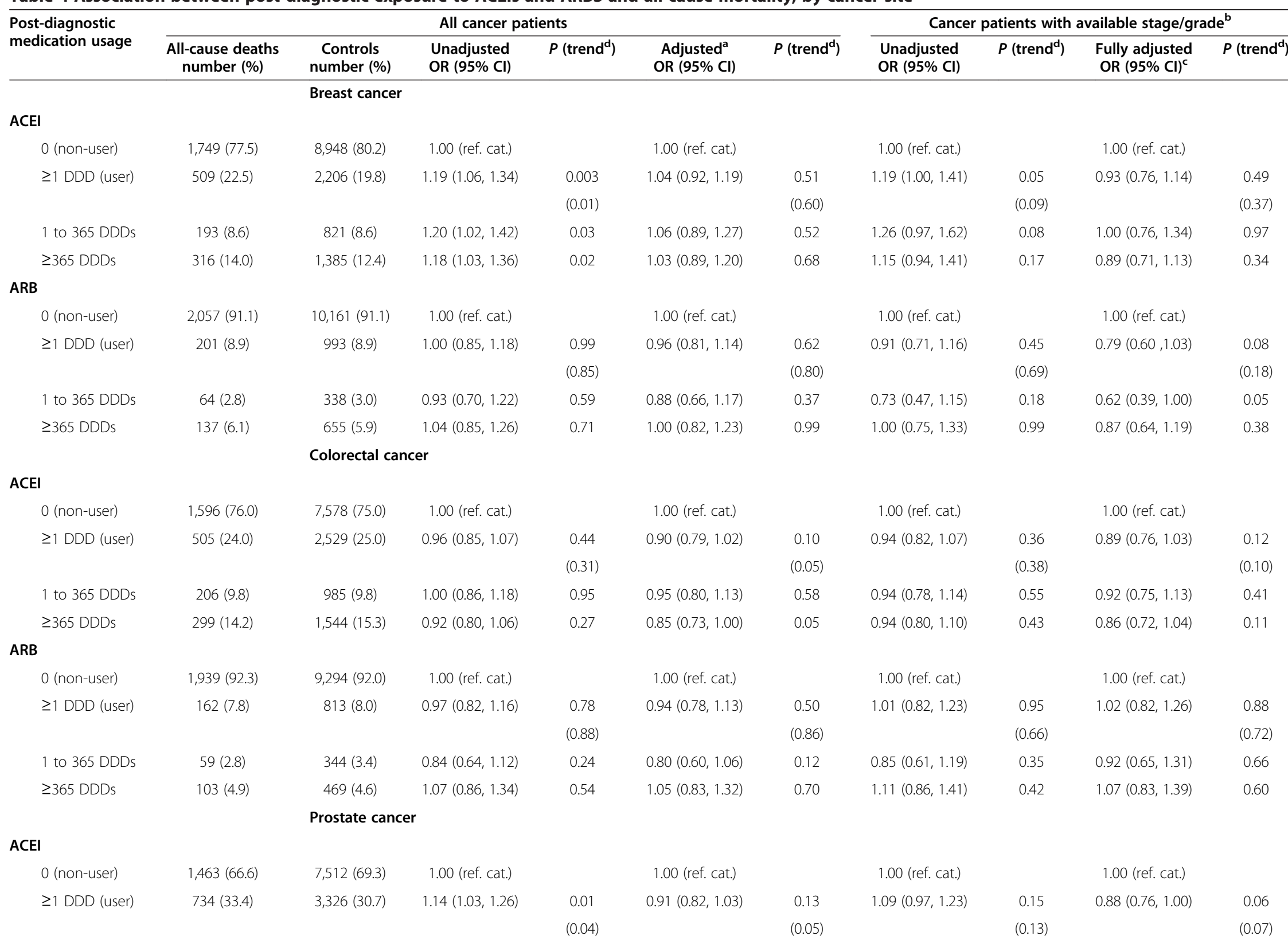


Table 4 Association between post-diagnostic exposure to ACEls and ARBS and all-cause mortality, by cancer site (Continued)

\begin{tabular}{|c|c|c|c|c|c|c|c|c|c|c|}
\hline 1 to 365 DDDs & $258(11.7)$ & $1,098(10.1)$ & $1.20(1.04,1.40)$ & 0.01 & $1.00(0.85,1.17)$ & 0.96 & $1.06(0.89,1.27)$ & 0.53 & $0.88(0.73,1.07)$ & 0.20 \\
\hline$\geq 365$ DDDs & $476(21.7)$ & $2,228(20.6)$ & $1.11(0.98,1.24)$ & 0.09 & $0.87(0.76,0.99)$ & 0.04 & $1.11(0.97,1.27)$ & 0.14 & $0.87(0.75,1.02)$ & 0.09 \\
\hline \multicolumn{11}{|l|}{ ARB } \\
\hline 0 (non-user) & $2,002(91.1)$ & $9,873(91.1)$ & 1.00 (ref. cat.) & & 1.00 (ref. cat.) & & 1.00 (ref. cat.) & & 1.00 (ref. cat.) & \\
\hline \multirow[t]{2}{*}{$\geq 1 \mathrm{DDD}$ (user) } & $195(8.9)$ & $965(8.9)$ & $1.00(0.85,1.18)$ & 0.98 & $0.85(0.72,1.01)$ & 0.06 & $1.08(0.89,1.30)$ & 0.45 & $0.92(0.75,1.12)$ & 0.39 \\
\hline & & & & (0.99) & & $(0.07)$ & & $(0.33)$ & & $(0.56)$ \\
\hline 1 to 365 DDDs & $61(2.8)$ & $297(2.7)$ & $1.02(0.77,1.35)$ & 0.89 & $0.86(0.64,1.15)$ & 0.32 & $0.95(0.68,1.33)$ & 0.77 & $0.81(0.57,1.14)$ & 0.26 \\
\hline$\geq 365$ DDDs & $134(6.1)$ & $668(6.2)$ & $0.99(0.82,1.21)$ & 0.95 & $0.84(0.69,1.03)$ & 0.10 & $1.14(0.91,1.43)$ & 0.26 & $0.97(0.76,1.23)$ & 0.81 \\
\hline
\end{tabular}

${ }^{\mathrm{a}}$ All models include surgery (within six months of diagnosis), chemotherapy (within six months), radiotherapy (within six months), low dose aspirin (during exposure period), statins (during exposure period), comorbidities (pre-diagnosis or during exposure period, including myocardial infarction, cerebrovascular disease, congestive heart disease, chronic pulmonary disease, peripheral vascular disease, renal disease, peptic ulcer disease and diabetes), and smoking (pre-diagnosis, with missing included as a category). The breast cancer model additionally includes tamoxifen (within six months), aromatase inhibitors (within six months), hormone replacement therapy (pre-diagnosis). The prostate cancer model additionally includes androgen therapy (within six months). ${ }^{\mathrm{b}}$ Analysis includes 1,558 cases and 7,406 controls in colorectal cancer patients with available stage, 1,008 cases and 4,900 controls in breast cancer patients with available stage and 1,520 cases and 7,473 controls in prostate cancer patients with available grade. ${ }^{\mathrm{C}} \mathrm{Additionally}$ adjusted for stage in colorectal cancer patients and breast cancer patients and adjusted for grade in prostate cancer patient. ${ }^{\mathrm{d}}$ Tests for trend from conditional logistic regression model based upon categories of DDDs $(0,1$ to $365, \geq 365$ DDDs coded as 0, 1, 2 respectively). ACEls, angiotensin-converting enzyme inhibitors; ARBs, angiotensin II receptor blockers; Cl, confidence interval; DDDs, daily defined doses; OR, odds ratio. 
Table 5 Sensitivity analyses for association between ACEls and ARBS and cancer specific mortality in colorectal cancer patients

\begin{tabular}{|c|c|c|c|c|c|c|}
\hline & \multirow{2}{*}{$\begin{array}{l}\text { Cancer specific } \\
\text { deaths number }\end{array}$} & \multirow{2}{*}{$\begin{array}{l}\text { Controls } \\
\text { number }\end{array}$} & \multirow{2}{*}{ 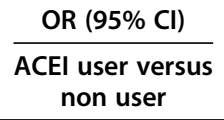 } & \multirow[t]{2}{*}{$P$} & \multirow{2}{*}{ 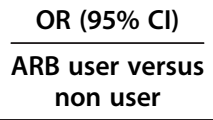 } & \multirow[t]{2}{*}{$P$} \\
\hline & & & & & & \\
\hline \multicolumn{7}{|l|}{ Colorectal cancer } \\
\hline Main analysis: diagnosis to six months prior to death ${ }^{a}$ & 1,093 & 5,231 & $0.76(0.62,0.93)$ & 0.01 & $0.80(0.59,1.09)$ & 0.16 \\
\hline Diagnosis to one year prior to death ${ }^{\mathrm{b}}$ & 869 & 4,152 & $0.83(0.67,1.03)$ & 0.10 & $0.89(0.64,1.25)$ & 0.51 \\
\hline $\begin{array}{l}\text { Restricted to users of any antihypertensive medication }{ }^{c} \text { prior } \\
\text { to cancer diagnosis }\end{array}$ & 427 & 1,934 & $0.83(0.65,1.07)$ & 0.15 & $0.90(0.63,1.27)$ & 0.54 \\
\hline $\begin{array}{l}\text { Comparison group restricted to users of any antihypertensive } \\
\text { in exposure period }^{c}\end{array}$ & 596 & 1,845 & $0.75(0.61,0.92)$ & 0.01 & $0.80(0.59,1.10)$ & 0.16 \\
\hline Additionally adjusting for other antihypertensives ${ }^{e}$ & 1,093 & 5,231 & $0.75(0.61,0.92)$ & 0.01 & $0.80(0.59,1.09)$ & 0.16 \\
\hline 2730 DDDs versus 0 DDDs (non-user) & 1,093 & 5,231 & $0.73(0.62,1.00)$ & 0.03 & $0.93(0.64,1.36)$ & 0.72 \\
\hline Stage 1 and $2^{f}$ & 328 & 1,483 & $0.54(0.38,0.77)$ & 0.001 & $0.80(0.47,1.37)$ & 0.41 \\
\hline Stage 3 and $4^{f}$ & 740 & 3,185 & $0.86(0.68,1.10)$ & 0.23 & $1.05(0.72,1.55)$ & 0.79 \\
\hline Colon & 649 & 3,134 & $0.84(0.64,1.09)$ & 0.19 & $0.77(0.52,1.15)$ & 0.21 \\
\hline Rectal & 444 & 2,097 & $0.67(0.49,0.92)$ & 0.01 & $0.91(0.56,1.50)$ & 0.72 \\
\hline Males & 638 & 3,078 & $0.80(0.62,1.03)$ & 0.08 & $0.93(0.62,1.41)$ & 0.74 \\
\hline Females & 455 & 2,153 & $0.70(0.49,0.98)$ & 0.04 & $0.64(0.40,1.03)$ & 0.06 \\
\hline Pre-diagnostic use $\mathrm{e}^{\mathrm{g}}$ & 1,641 & 7,815 & $1.06(0.89,1.27)$ & 0.51 & $0.86(0.62,1.18)$ & 0.35 \\
\hline Time varying covariate analysis ${ }^{h}$ & 1,109 & 2,559 & & & & \\
\hline$\geq 1$ DDDs versus 0 DDDs (non-user) & & & $0.81(0.69,0.96)$ & 0.01 & $0.86(0.66,1.12)$ & 0.26 \\
\hline 1 to365 DDDs versus 0 DDDs (non-user) & & & $0.76(0.59,0.97)$ & 0.03 & $0.64(0.40,1.03)$ & 0.07 \\
\hline 2365 DDDs versus 0 DDDs (non-user) & & & $0.85(0.69,1.03)$ & 0.10 & $1.00(0.73,1.38)$ & 0.99 \\
\hline$\geq 1$ DDDs versus 0 DDDs (non-user) in stage 1 and 2 patients & & & $0.62(0.46,0.84)$ & 0.002 & $0.65(0.41,1.05)$ & 0.08 \\
\hline$\geq 1$ DDDs versus 0 DDDs (non-user) in stage 3 and 4 patients & & & $0.91(0.74,1.10)$ & 0.33 & $0.97(0.70,1.34)$ & 0.83 \\
\hline
\end{tabular}

${ }^{a}$ Except where otherwise stated analysis investigates medications from diagnosis to six months prior to death/index date and models include surgery (within six months of diagnosis), chemotherapy (within six months), radiotherapy (within six months), low dose aspirin (during exposure period), statins (during exposure period), comorbidities (pre-diagnosis or during exposure period, including myocardial infarction, cerebrovascular disease, congestive heart disease, chronic pulmonary disease, peripheral vascular disease, renal disease, peptic ulcer disease and diabetes), smoking (pre-diagnosis, with missing included as a category) and stage. ${ }^{b}$ Restricted to individuals surviving more than 1.5 years. 'Antihypertensive medications include beta-blockers, diuretics, vasodilator antihypertensive drugs, centrally acting antihypertensive drugs, alpha-adrenoceptor blocking drugs, ACEls, ARBs, renin inhibitors and calcium channel blockers. ${ }^{\mathrm{d}}$ Restricted to individuals with $>1$ year of records prior to cancer diagnosis and pre-diagnostic use considered antihypertensive use in that year, excluding deaths in the year after cancer diagnosis. ${ }^{e}$ Models include all variables in ${ }^{a}$ and additionally include calcium channel blockers, diuretics and beta-blockers. ${ }^{\mathrm{f}}$ Stratified analyses were conducted after re-matching controls to cases within strata, due to non-availability of matches overall numbers in subgroups may not be identical to numbers presented in Table 1. ${ }^{9}$ Restricted to individuals with $>1$ year of records prior to cancer diagnosis and pre-diagnostic use considered ACEI/ARB use in that year, not excluding deaths in the year after cancer diagnosis. " Reported estimates are adjusted hazard ratios and $95 \% \mathrm{Cl}$, model contains age at diagnosis, year of diagnosis, gender, site (colon or rectum), surgery (within six months of diagnosis), chemotherapy (within six months), radiotherapy (within six months), statins (during exposure period as a time varying covariate), comorbidities (pre-diagnosis including myocardial infarction, cerebrovascular disease, congestive heart disease, chronic pulmonary disease, peripheral vascular disease, renal disease, peptic ulcer disease and diabetes) and stage. ACEls, angiotensin-converting enzyme inhibitors; ARBs, angiotensin II receptor blockers; $\mathrm{Cl}$, confidence interval; DDDs, daily defined doses; OR, odds ratio.

ACEI and cancer progression, one [17] in prostate cancer patients and two $[15,16]$ in colorectal cancer patients. An earlier ACEI study in 62 prostate cancer patients after radical prostatectomy [17] observed a reduced rate of biochemical recurrence in ACEI users compared with non-users (3/32 versus 10/30). An earlier ACEI study in 55 stage 2 colorectal cancer patients [15] observed a reduction in the risk of distant metastasis with frequent ACEI use prior to diagnosis (adjusted $\mathrm{OR}=0.22$ ) and a more recent study in 262 stage 3 and 4 cancer patients did not present an estimate for ACEI or ARB use separately but did present a reduced risk of mortality in patients simultaneously using beta-blockers and ACEIs or ARBs ( $\mathrm{HR}=0.5095 \%$ CI 0.29, 0.85).

Five independent epidemiological studies [13,14,18-20] have previously investigated ACEI and cancer-specific mortality in breast cancer patients but only two have previously investigated ARB use separately. A recent large Danish study [13] demonstrated a small but non-significant increase in cancer recurrence with ACEI use but no association with $\mathrm{ARB}$ use $(\mathrm{HR}=1.295 \%$ CI 0.97, 1.4 and $\mathrm{HR}=1.195 \%$ CI 0.85, 1.3, respectively). Another recent US study [14], from the same cohort as an earlier study [34], observed no association between either ACEI use 
Table 6 Sensitivity analyses for association between ACEIs and ARBS and cancer specific mortality in prostate cancer patients

\begin{tabular}{|c|c|c|c|c|c|c|}
\hline & Cancer specific & Controls & OR $(95 \% \mathrm{Cl})$ & $P$ & OR $(95 \% \mathrm{Cl})$ & $\mathbf{P}$ \\
\hline & & & $\begin{array}{l}\text { ACEI user versus } \\
\text { non user }\end{array}$ & & $\begin{array}{l}\text { ARB user versus } \\
\text { non user }\end{array}$ & \\
\hline Prostate cancer & & & & & & \\
\hline Main analysis: diagnosis to six months prior to death ${ }^{\mathrm{a}}$ & 766 & 3,777 & $0.82(0.67,1.02)$ & 0.07 & $0.82(0.61,1.11)$ & 0.21 \\
\hline Diagnosis to one year prior to death ${ }^{b}$ & 682 & 3,360 & $0.88(0.70,1.09)$ & 0.24 & $0.89(0.64,1.23)$ & 0.48 \\
\hline Adjusting for Gleason score along with all variables in ${ }^{a}$ apart from grade & 516 & 2,518 & $0.80(0.62,1.03)$ & 0.09 & $0.89(0.61,1.31)$ & 0.55 \\
\hline $\begin{array}{l}\text { Restricted to users of any antihypertensive medication }{ }^{c} \text { prior } \\
\text { to cancer diagnosis }\end{array}$ & 399 & 1,925 & $0.80(0.62,1.04)$ & 0.09 & $0.89(0.62,1.26)$ & 0.51 \\
\hline $\begin{array}{l}\text { Comparison group restricted to users of any antihypertensive in } \\
\text { exposure period }\end{array}$ & 584 & 2,753 & $0.78(0.63,0.96)$ & 0.02 & $0.80(0.59,1.08)$ & 0.15 \\
\hline Additionally adjusting for other antihypertensives ${ }^{\mathrm{e}}$ & 766 & 3,777 & $0.74(0.60,0.92)$ & 0.01 & $0.75(0.55,1.02)$ & 0.07 \\
\hline Additionally adjusting for hormone therapy any time after diagnosis ${ }^{f}$ & 766 & 3,777 & $0.82(0.66,1.01)$ & 0.06 & $0.84(0.62,1.14)$ & 0.27 \\
\hline$\geq 730$ DDDs versus 0 DDDs (non-user) & 766 & 3,777 & $0.91(0.70,1.18)$ & 0.49 & $0.84(0.59,1.21)$ & 0.36 \\
\hline Pre-diagnostic use ${ }^{\mathrm{g}}$ & 821 & 4,041 & $0.98(0.78,1.23)$ & 0.87 & $1.14(0.75,1.75)$ & 0.53 \\
\hline Time varying covariate analysis ${ }^{\mathrm{h}}$ & 772 & 3,838 & & & & \\
\hline$\geq 1$ DDDs versus 0 DDDs (non-user) & & & $0.90(0.76,1.07)$ & 0.24 & $0.95(0.73,1.23)$ & 0.70 \\
\hline 1 to 365 DDDs versus 0 DDDs (non-user) & & & $0.87(0.70,1.08)$ & 0.21 & $0.88(0.62,1.26)$ & 0.49 \\
\hline$\geq 365$ DDDs versus 0 DDDs (non-user) & & & $0.94(0.76,1.18)$ & 0.60 & $1.03(0.72,1.48)$ & 0.86 \\
\hline
\end{tabular}

${ }^{a}$ Except where otherwise stated analysis investigates medications from diagnosis to six months prior to death/index date and models include surgery (within six months of diagnosis), chemotherapy (within six months), radiotherapy (within six months), low dose aspirin (during exposure period), statins (during exposure period), comorbidities (pre-diagnosis or during exposure period, including myocardial infarction, cerebrovascular disease, congestive heart disease, chronic pulmonary disease, peripheral vascular disease, renal disease, peptic ulcer disease and diabetes), smoking (pre-diagnosis, with missing included as a category), androgen therapy (within six months) and grade. ${ }^{b}$ Restricted to individuals surviving more than 1.5 years. 'Antihypertensive medications include beta-blockers, diuretics, vasodilator antihypertensive drugs, centrally acting antihypertensive drugs, alpha-adrenoceptor blocking drugs, ACEls, ARBs, renin inhibitors, and calcium channel blockers. ${ }^{\mathrm{d}}$ Restricted to individuals with $>1$ year of records prior to cancer diagnosis and pre-diagnostic use considered antihypertensive use in that year, excluding deaths in the year after cancer diagnosis.

${ }^{\mathrm{e}}$ Models include all variables in and additionally include calcium channel blockers, diuretics and beta-blockers. ${ }^{\mathrm{f}}$ Models include all variables in ${ }^{\mathrm{a}}$ but androgen therapy was determined at any time after diagnosis in the exposure period. ${ }^{9}$ Restricted to individuals with $>1$ year of records prior to cancer diagnosis and

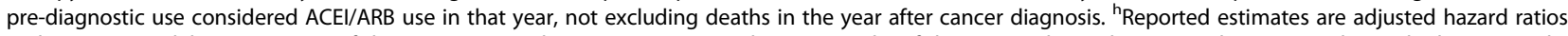
and $95 \%$ Cls models contain year of diagnosis, age at diagnosis, surgery (within six months of diagnosis), chemotherapy (within six months), radiotherapy (within six months), comorbidities (pre-diagnosis, including myocardial infarction, cerebrovascular disease, congestive heart disease, chronic pulmonary disease, peripheral vascular disease, renal disease, peptic ulcer disease and diabetes), statin (as a time varying covariate) and grade. ACEls, angiotensin-converting enzyme inhibitors; ARBs, angiotensin II receptor blockers; $\mathrm{Cl}$, confidence interval; DDDs, daily defined doses; OR, odds ratio.

$(\mathrm{HR}=1.0795 \%$ CI $0.65,1.77)$ or $\mathrm{ARB}$ use $(\mathrm{HR}=0.41$ 95\% CI $0.15,1.13$ ) and cancer-specific mortality. An earlier smaller study [18], including 174 breast cancer-specific deaths in 1,779 breast cancer patients, observed no association with simultaneous ACEI and beta-blocker use and when investigating ACEI users exclusively observed a marked increase in cancer recurrence $(\mathrm{HR}=1.5695 \%$ CI 1.02, 2.39) but little evidence of an increase in breast cancer-specific mortality ( $\mathrm{HR}=1.2795 \% \mathrm{CI} 0.74,2.19)$. One study observed little evidence of association for self-reported ACEI use ( $\mathrm{HR}=0.89$ 95\% CI 0.60, 1.32) [20]. Finally another study observed a marked reduction in cancer recurrence in 168 stage 2 or 3 breast cancer patients using ACEI or ARBs after diagnosis $(\mathrm{HR}=0.57$ 95\% CI $0.37,0.89, P=0.01$ ) [19], but this estimate may have incurred some immortal time bias [29] as individuals using ACEIs or ARBs after diagnosis were considered users from diagnosis in the analysis so in the period from diagnosis to medication use they could not have died.
Our study observed some protective effects of ACEIs, particularly in early stage colorectal cancer patients and prostate cancer patients, which, if real, would support the theory that ACEIs have a role in cancer therapy [35,36]. Components of the renin-angiotensin system (RAS) are expressed in various cancer sites [37] and may contribute to processes important for cancer progression including cell proliferation and apoptosis. In vitro and animal studies have shown that both ACEIs and ARBs can suppress cell proliferation and tumor/metastasis growth in various cancers including breast [38] and colorectal [39]. Angiogenesis also appears to be an important process by which the RAS system exerts pro-tumor effects and ACEIs and ARBs reduce the expression of vascular endothelial growth factor (VEGF) and other angiogenic factors in both cell lines [38] and animal models [40]. However, the protective effects observed in colorectal and prostate cancer patients should be interpreted cautiously because, as previously stated, the effects were weak, inconsistent and not a priori stated. 


\section{Conclusions}

In conclusion, concerns about the safety of ACEIs and ARBs in cancer patients have been raised by trial data showing increases in fatal cancers in ARB users [4] and observational data showing increases in breast cancer recurrence rates in ACEI users [18]. In contrast, our study provides no evidence of increased risks of cancer-specific mortality in users of ACEIs or ARBs with breast, prostate or colorectal cancer and suggests that these medications are safe in patients diagnosed with these common cancers.

\section{Competing interests}

The authors declare that they have no competing interests.

\section{Authors' contributions}

Study concept and design: CRC, CH, LJM. Acquisition of data: CRC, UCM, CH, MMC, LJM. Analysis and interpretation of data: all authors. Drafting of the manuscript: CRC. Critical revision of the manuscript for important intellectual content: all authors. Statistical analysis: CRC, UCM, BMH. Obtained funding: CRC, CH, LJM. All authors read and approved the final manuscript.

\section{Acknowledgements}

CRC was supported by a Health and Social Care Research and Development, Public Health Agency, Northern Ireland, funded UK NIHR awarded Career Development Fellowship. BMH and UCM were funded by Northern Ireland Department of Education and Learning PhD studentships. Access to the GPRD database was funded through the Medical Research Council's license agreement with the UK Medicines and Healthcare Products Regulatory Agency. However, the interpretation and conclusions contained in this study are those of the authors alone. None of the funders had any role in the design and conduct of the study; collection, management, analysis, and interpretation of the data; and preparation, review, or approval of the manuscript.

\section{Author details}

${ }^{1}$ Cancer Epidemiology and Health Services Research Group, Centre for Public Health, Queen's University Belfast, Belfast, Northern Ireland. ${ }^{2}$ School of Pharmacy, Queen's University Belfast, Belfast, Northern Ireland.

Received: 21 August 2013 Accepted: 14 January 2014 Published: 13 February 2014

\section{References}

1. Maddams J, Utley M, Moller $\mathrm{H}$ : Projections of cancer prevalence in the United Kingdom, 2010-2040. Br J Cancer 2012, 107:1195-1202.

2. de Moor JS, Mariotto AB, Parry C, Alfano CM, Padgett L, Kent EE, Forsythe L, Scoppa S, Hachey M, Rowland JH: Cancer survivors in the United States: prevalence across the survivorship trajectory and implications for care. Cancer Epidemiol Biomarkers Prev 2013, 22:561-570.

3. Piccirillo JF, Tierney RM, Costas I, Grove L, Spitznagel EL: Prognostic importance of comorbidity in a hospital-based cancer registry. JAMA 2004, 291:2441-2447.

4. Pfeffer MA, Swedberg K, Granger CB, Held P, McMurray JJ, Michelson EL, Olofsson B, Ostergren J, Yusuf S, Pocock S: Effects of candesartan on mortality and morbidity in patients with chronic heart failure: the CHARM-Overall programme. Lancet 2003, 362:759-766.

5. Sipahi I, Debanne SM, Rowland DY, Simon DI, Fang JC: Angiotensin-receptor blockade and risk of cancer: meta-analysis of randomised controlled trials. Lancet Oncol 2010, 11:627-636.

6. Goldstein MR, Mascitelli L, Pezzetta F: Angiotensin-receptor blockade, cancer, and concerns. Lancet Oncol 2010, 11:817-818.

7. Clere N, Corre I, Faure S, Guihot AL, Vessieres E, Chalopin M, Morel A, Coqueret O, Hein L, Delneste Y, Paris F, Henrion D: Deficiency or blockade of angiotensin II type 2 receptor delays tumorigenesis by inhibiting malignant cell proliferation and angiogenesis. Int J Cancer 2010, 127:2279-2291.

8. Bangalore S, Kumar S, Kjeldsen SE, Makani H, Grossman E, Wetterslev J, Gupta AK, Sever PS, Gluud C, Messerli FH: Antihypertensive drugs and risk of cancer: network meta-analyses and trial sequential analyses of 324,168 participants from randomised trials. Lancet Oncol 2011, 12:65-82.

9. Hallas J, Christensen R, Andersen M, Friis S, Bjerrum L: Long term use of drugs affecting the renin-angiotensin system and the risk of cancer: a populationbased case-control study. Br J Clin Pharmacol 2012, 74:180-188.

10. Pasternak B, Svanstrom H, Callreus T, Melbye M, Hviid A: Use of angiotensin receptor blockers and the risk of cancer. Circulation 2011, 123:1729-1736.

11. Azoulay L, Assimes TL, Yin H, Bartels DB, Schiffrin EL, Suissa S: Long-term use of angiotensin receptor blockers and the risk of cancer. PLOS One 2012, 7:e50893.

12. Bhaskaran K, Douglas I, Evans S, van Staa T, Smeeth L: Angiotensin receptor blockers and risk of cancer: cohort study among people receiving antihypertensive drugs in UK General Practice Research Database. BMJ 2012, 344:e2697.

13. Sorensen GV, Ganz PA, Cole SW, Pedersen LA, Sorensen HT, Cronin-Fenton DP, Game JP, Christiansen PM, Lash TL, Ahern TP: Use of $\beta$-blockers, angiotensin-converting enzyme inhibitors, angiotensin II receptor blockers, and risk of breast cancer recurrence: a Danish nationwide prospective cohort study. J Clin Oncol 2013, 31:2265-2272.

14. Chae YK, Brown EN, Lei X, Melhem-Bertrandt A, Giordano SH, Litton JK, Hortobagyi GN, Gonzalez-Angulo AM, Chavez-Macgregor M: Use of ACE inhibitors and angiotensin receptor blockers and primary breast cancer outcomes. J Cancer 2013, 4:549-556.

15. Heinzerling $\mathrm{JH}$, Anthony T, Livingston EH, Huerta S: Predictors of distant metastasis and mortality in patients with stage II colorectal cancer. Am Surg 2007, 73:230-238

16. Engineer DR, Burney BO, Hayes TG, Garcia JM: Exposure to ACEl/ARB and beta-blockers is associated with improved survival and decreased tumor progression and hospitalizations in patients with advanced colon cancer. Transl Oncol 2013, 6:539-545.

17. Ronquist G, Frithz G, Wang YH, Lindeborg T: Captopril may reduce biochemical (prostate-specific antigen) failure following radical prostatectomy for clinically localized prostate cancer. Scand J Urol Nephrol 2009, 43:32-36.

18. Ganz PA, Habel LA, Weltzien EK, Caan BJ, Cole SW: Examining the influence of beta blockers and ACE inhibitors on the risk for breast cancer recurrence: results from the LACE cohort. Breast Cancer Res Treat 2011, 129:549-556.

19. Chae YK, Valsecchi ME, Kim J, Bianchi AL, Khemasuwan D, Desai A, Tester W: Reduced risk of breast cancer recurrence in patients using ACE inhibitors, ARBs, and/or statins. Cancer Invest 2011, 29:585-593.

20. Holmes MD, Hankinson SE, Feskanich D, Chen WY: Beta blockers and angiotensin-converting enzyme inhibitors' purported benefit on breast cancer survival may be explained by aspirin use. Breast Cancer Res Treat 2013, 139:507-513.

21. Boggon R, van Staa TP, Chapman M, Gallagher AM, Hammad TA, Richards MA: Cancer recording and mortality in the General Practice Research Database and linked cancer registries. Pharmacoepidemiol Drug Saf 2013, 22:168-175.

22. Herrett E, Thomas SL, Schoonen WM, Smeeth L, Hall AJ: Validation and validity of diagnoses in the General Practice Research Database: a systematic review. Br J Clin Pharmacol 2010, 69:4-14.

23. Clinical Practice Research Datalink: Ethical review for CPRD protocols. [http://www.cprd.com/isac/otherinfo.asp]

24. British Medical Association and the Royal Pharmaceutical Society of Great Britain: British National Formulary. [http://www.bnf.org/bnf/index.htm]

25. World Health Organisation: World Health Organisation Collaborating Centre for Drug Statistics Methodology. [http://www.whocc.no/]

26. National Cancer Institute: Prostate cancer treatment. [http://www.cancer. gov/cancertopics/pdq/treatment/prostate/HealthProfessional/page2]

27. Khan NF, Perera R, Harper S, Rose PW: Adaptation and validation of the Charlson Index for Read/OXMIS coded databases. BMC Fam Pract 2010, 11:1

28. Schade R, Andersohn F, Suissa S, Haverkamp W, Garbe E: Dopamine agonists and the risk of cardiac-valve regurgitation. N Engl J Med 2007, 356:29-38.

29. Levesque LE, Hanley JA, Kezouh A, Suissa S: Problem of immortal time bias in cohort studies: example using statins for preventing progression of diabetes. BMJ 2010, 340:b5087.

30. Etminan M: Pharmacoepidemiology II: the nested case-control study-a novel approach in pharmacoepidemiologic research. Pharmacotherapy 2004, 24:1105-1109. 
31. Essebag V, Platt RW, Abrahamowicz M, Pilote L: Comparison of nested case-control and survival analysis methodologies for analysis of time-dependent exposure. BMC Med Res Methodol 2005, 5:5.

32. Altman DG, Bland JM: Interaction revisited: the difference between two estimates. BMJ 2003, 326:219.

33. Fine JP, Gray RJ: A proportional hazards model for the subdistribution of a competing risk. J Am Stat Assoc 1999, 94:496-509.

34. Melhem-Bertrandt A, Chavez-Macgregor M, Lei X, Brown EN, Lee RT, Meric-Bernstam F, Sood AK, Conzen SD, Hortobagyi GN, Gonzalez-Angulo AM: Beta-blocker use is associated with improved relapse-free survival in patients with triple-negative breast cancer. J Clin Oncol 2011, 29:2645-2652.

35. Ager El, Neo J, Christophi C: The renin-angiotensin system and malignancy. Carcinogenesis 2008, 29:1675-1684

36. George AJ, Thomas WG, Hannan RD: The renin-angiotensin system and cancer: old dog, new tricks. Nat Rev Cancer 2010, 10:745-759.

37. Deshayes F, Nahmias C: Angiotensin receptors: a new role in cancer? Trends Endocrinol Metab 2005, 16:293-299.

38. Chen X, Meng Q, Zhao Y, Liu M, Li D, Yang Y, Sun L, Sui G, Cai L, Dong X: Angiotensin II type 1 receptor antagonists inhibit cell proliferation and angiogenesis in breast cancer. Cancer Lett 2013, 328:318-324.

39. Neo JH, Malcontenti-Wilson C, Muralidharan V, Christophi C: Effect of ACE inhibitors and angiotensin II receptor antagonists in a mouse model of colorectal cancer liver metastases. J Gastroenterol Hepatol 2007, 22:577-584.

40. Wang L, Cai SR, Zhang CH, He YL, Zhan WH, Wu H, Peng JJ: Effects of angiotensin-converting enzyme inhibitors and angiotensin II type 1 receptor blockers on lymphangiogenesis of gastric cancer in a nude mouse model. Chin Med J (Engl) 2008, 121:2167-2171.

doi:10.1186/1741-7015-12-28

Cite this article as: Cardwell et al:: Drugs affecting the renin-angiotensin system and survival from cancer: a population based study of breast colorectal and prostate cancer patient cohorts. BMC Medicine 2014 12:28.

\section{Submit your next manuscript to BioMed Central and take full advantage of:}

- Convenient online submission

- Thorough peer review

- No space constraints or color figure charges

- Immediate publication on acceptance

- Inclusion in PubMed, CAS, Scopus and Google Scholar

- Research which is freely available for redistribution 